\title{
On the Non-Self-adjoint Sturm-Liouville Operators in the Space of Vector-Functions
}

\author{
Fulya Şeref and O. A. Veliev \\ Department of Mathematics, Dogus University, Kadiköy, 34722, \\ Istanbul, Turkey. E-mail: fseref@dogus.edu.tr; oveliev@dogus.edu.tr
}

\begin{abstract}
In this article we obtain asymptotic formulas for the eigenvalues and eigenfunctions of the non-self-adjoint operator generated in $L_{2}^{m}[0,1]$ by the Sturm-Liouville equation with $m \times m$ matrix potential and the boundary conditions whose scalar case $(m=1)$ are strongly regular. Using these asymptotic formulas, we find a condition on the potential for which the root functions of this operator form a Riesz basis.

AMS Mathematics Subject Classification numbers: 34L10, $47 \mathrm{E} 05$.

Keywords: Differential operators, matrix potential, Riesz basis.
\end{abstract}

\section{Introduction and Preliminary Facts}

We consider the differential operator $L_{m}(Q)$ generated in the space $L_{2}^{m}[0,1]$ by the differential expression

$$
-\mathbf{y}^{\prime \prime}(x)+Q(x) \mathbf{y}(x)
$$

and the boundary conditions whose scalar case (the case $m=1$ ) are strongly regular, where $\mathbf{y}(x)=\left(y_{1}(x), y_{2}(x), \ldots, y_{m}(x)\right)^{T}, L_{2}^{m}[0,1]$ is the set of vectorfunctions $\mathbf{f}(x)=\left(f_{1}(x), f_{2}(x), \ldots, f_{m}(x)\right)$ with $f_{k} \in L_{2}[0,1]$ for $k=1,2, \ldots, m$ and $Q(x)=\left(b_{i, j}(x)\right)$ is a $m \times m$ matrix with the complex-valued square integrable entries $b_{i, j}$. The norm $\|$.$\| and inner product (.,$.$) in L_{2}^{m}[0,1]$ are defined by

$$
\|f\|=\left(\int_{0}^{1}|f(x)|^{2} d x\right)^{\frac{1}{2}},(f, g)=\int_{0}^{1}\langle f(x), g(x)\rangle d x,
$$

where $|$.$| and \langle.,$.$\rangle are respectively the norm and the inner product in \mathbb{C}^{m}$.

A. A. Shkalikov [8-12] proved that the root functions (eigenfunctions and associated eigenfunctions) of the operators generated by an ordinary differential expression with summable matrix coefficients and regular boundary conditions form a Riesz basis with parenthesis and in parenthesis should be included only 
the functions corresponding to splitting eigenvalues. L. M. Luzhina [3] generalized this result for the boundary value problems when the coefficients depend on the spectral parameter.

In [13], the differential operator $T_{t}(Q)$ generated in the space $L_{2}^{m}[0,1]$ by the differential expression (1) and the quasiperiodic conditions

$$
\mathbf{y}^{\prime}(1)=e^{i t} \mathbf{y}^{\prime}(0), \mathbf{y}(1)=e^{i t} \mathbf{y}(0)
$$

for $t \in(0,2 \pi)$ and $t \neq \pi$ was considered . In [13], we proved that the eigenvalues $\lambda_{k, j}$ of $T_{t}(Q)$ lie in the $O\left(\frac{\ln k}{k}\right)$ neighborhoods of the eigenvalues of the operator $T_{t}(C)$, where

$$
C=\int_{0}^{1} Q(x) d x .
$$

Note that, to obtain the asymptotic formulas of order $O\left(\frac{1}{k}\right)$ for the eigenvalues $\lambda_{k, j}$ of the differential operators generated by (1), using the classical asymptotic expansions for the solutions of the matrix equation $-Y^{\prime \prime}+Q(x) Y=\lambda Y$, it is required that $Q$ be differentiable (see $[1,4,5,7]$ ). The suggested method in [13] gives the possibility of obtaining the asymptotic formulas of order $O\left(k^{-1} \ln |k|\right)$ for the eigenvalues $\lambda_{k, j}$ and the normalized eigenfunctions $\Psi_{k, j}(x)$ of $T_{t}(Q)$ when there is not any condition about smoothness of the entries $b_{i, j}$ of $Q$. Besides, in [13], using the asymptotic formulas, it was proved that if the eigenvalues of the matrix $C$ are simple, then the root functions of the operator $T_{t}(Q)$ form a Riesz basis. Then, in papers [14-17], using the method of [13], we considered the spectrum and basis property of the root functions of differential operators generated in $L_{2}^{m}[0,1]$ by the differential expression of arbitrary order and by the $t$-periodic, periodic, antiperiodic boundary conditions and applied these investigations to the differential operators with periodic matrix coefficients.

In this paper, we investigate the operator $L_{m}(Q)$ generated in $L_{2}^{m}[0,1]$ by (1) and the boundary conditions

$$
U_{i}(\mathbf{y})=\alpha_{i} \mathbf{y}^{\left(k_{i}\right)}(0)+\alpha_{i, 0} \mathbf{y}(0)+\beta_{i} \mathbf{y}^{\left(k_{i}\right)}(1)+\beta_{i, 0} \mathbf{y}(1)=0, \quad i=1,2
$$

whose scalar case (the case $m=1$ )

$$
U_{i}(y)=\alpha_{i} y^{\left(k_{i}\right)}(0)+\alpha_{i, 0} y(0)+\beta_{i} y^{\left(k_{i}\right)}(1)+\beta_{i, 0} y(1)=0, \quad i=1,2
$$

are strongly regular, where $0 \leq k_{2} \leq k_{1} \leq 1, \alpha_{i}, \alpha_{i, 0}, \beta_{i}, \beta_{i, 0}$ are complex numbers and for each value of the index $i$ at least one of the numbers $\alpha_{i}, \beta_{i}$ is nonzero.

Recall that the conditions (4) are called regular if the numbers $\theta_{-1}$ and $\theta_{1}$ defined by the identity

$$
\frac{\theta_{-1}}{s}+\theta_{0}+\theta_{1} s=\operatorname{det}\left[\begin{array}{cc}
\left(\alpha_{1}+s \beta_{1}\right) \omega_{1}^{k_{1}} & \left(\alpha_{1}+\frac{1}{s} \beta_{1}\right) \omega_{2}^{k_{1}} \\
\left(\alpha_{2}+s \beta_{2}\right) \omega_{1}^{k_{2}} & \left(\alpha_{2}+\frac{1}{s} \beta_{2}\right) \omega_{2}^{k_{2}}
\end{array}\right]
$$

are different from zero, where $\omega_{1}$ and $\omega_{2}$ are the distinct square roots of -1 (see [7] p. 57). The regular boundary conditions are said to be strongly regular if 
$\theta_{0}^{2}-4 \theta_{1} \theta_{-1} \neq 0$. If the boundary conditions (4) is regular then the equality (5) is either

$$
\frac{\theta_{-1}}{s}+\theta_{0}+\theta_{1} s=\frac{1}{s}-s
$$

or

$$
\frac{\theta_{-1}}{s}+\theta_{0}+\theta_{1} s=a\left(s+\frac{1}{s}\right)+b
$$

(see p. 63 of [7]), where $a, b$ are complex numbers and $a \neq 0$.

The eigenvalues of the operator $L_{1}(q)$ generated in the space $L_{2}[0,1]$ by the differential expression $-y^{\prime \prime}(x)+q(x) y(x)$ and strongly regular boundary conditions (4), where $q$ is a summable function, consist of the sequences $\left\{\rho_{n}^{(1)}(q)\right\}$ and $\left\{\rho_{n}^{(2)}(q)\right\}$ satisfying

$$
\rho_{n}^{(1)}(q)=\left(2 n \pi+\gamma_{1}\right)^{2}+O(1), \rho_{n}^{(2)}(q)=\left(2 n \pi+\gamma_{2}\right)^{2}+O(1) ; \quad n \geq N>>1,
$$

where

$$
\gamma_{j}=-i \ln \zeta_{j}, \operatorname{Re} \gamma_{j} \in(-\pi, \pi], \zeta_{1} \neq \zeta_{2}
$$

and $\zeta_{1}, \zeta_{2}$ are the roots of the equation

$$
\theta_{1} \zeta^{2}+\theta_{0} \zeta+\theta_{-1}=0
$$

(see [7], p. 65, formulas (45a), (45b)).

One can readily see that in case (6) we have

$$
\gamma_{1}=0, \gamma_{2}=\pi
$$

In case (7), equation (10) has the form

$$
\zeta^{2}+\frac{b}{a} \zeta+1=0
$$

that is, $\zeta_{1} \zeta_{2}=1$ and by (9) $\zeta_{1} \neq \zeta_{2}$ which implies that $\zeta_{1} \neq \pm 1$ and $\zeta_{2} \neq \pm 1$. Therefore, in case (7) we have

$$
\gamma_{1}=-\gamma_{2} \neq \pi k
$$

In this paper, first we prove that if the boundary conditions (4) are regular, then the boundary conditions (3) are also regular (see Theorem 1$)$. Then, as in [13], we consider the operator $L_{m}(Q)$ as perturbation of $L_{m}(C)$ by $Q-C$ and obtain asymptotic formulas for the eigenvalues and eigenfunctions of $L_{m}(Q)$ in term of the eigenvalues and eigenfunctions of $L_{m}(C)$, where $C$ is defined in (2). Finally, using the obtained asymptotic formulas and the theorem of Bari (see p.310 of [2]), we prove that if the eigenvalues of the matrix $C$ are simple, then the root functions of the operator $L_{m}(Q)$ form a Riesz basis. 


\section{Main Results}

First, let us consider the boundary conditions (3).

Theorem 1 If the boundary conditions (4) are regular then the boundary conditions (3) are also regular.

Proof. The conditions (3) are regular (see [7], p. 121) if the numbers $\Theta_{-m}$ , $\Theta_{m}$ defined by the identity

$$
\Theta_{-m} s^{-m}+\Theta_{-m+1} s^{m-1}+\ldots+\Theta_{m} s^{m}=\operatorname{det} M(m)
$$

are both different from zero, where

$$
M(m)=\left[\begin{array}{ll}
\left(\alpha_{1}+s \beta_{1}\right) \omega_{1}^{k_{1}} I & \left(\alpha_{1}+\frac{1}{s} \beta_{1}\right) \omega_{2}^{k_{1}} I \\
\left(\alpha_{2}+s \beta_{2}\right) \omega_{1}^{k_{2}} I & \left(\alpha_{2}+\frac{1}{s} \beta_{2}\right) \omega_{2}^{k_{2}} I
\end{array}\right]
$$

and $I$ is $m \times m$ identity matrix. One can easily see that the intersection of the first and $(m+1)$-th rows and columns forms the matrix

$$
M(1)=\left[\begin{array}{cc}
\left(\alpha_{1}+s \beta_{1}\right) \omega_{1}^{k_{1}} & \left(\alpha_{1}+\frac{1}{s} \beta_{1}\right) \omega_{2}^{k_{1}} \\
\left(\alpha_{2}+s \beta_{2}\right) \omega_{1}^{k_{2}} & \left(\alpha_{2}+\frac{1}{s} \beta_{2}\right) \omega_{2}^{k_{2}}
\end{array}\right]
$$

and its complementary minor is $M(m-1)$. Moreover, the determinant of the minors of $M(m)$ formed by intersection of the first and $(m+1)$-th rows and other pairs of columns is zero, since the $2 \times m$ matrix consisting of these rows has the form

$$
\left[\begin{array}{cccccccccc}
\left(\alpha_{1}+s \beta_{1}\right) \omega_{1}^{k_{1}} & 0 & 0 & \ldots & 0 & \left(\alpha_{1}+\frac{1}{s} \beta_{1}\right) \omega_{2}^{k_{1}} & 0 & 0 & \ldots & 0 \\
\left(\alpha_{2}+s \beta_{2}\right) \omega_{1}^{k_{2}} & 0 & 0 & \ldots & 0 & \left(\alpha_{2}+\frac{1}{s} \beta_{2}\right) \omega_{2}^{k_{2}} & 0 & 0 & \ldots & 0
\end{array}\right] .
$$

Therefore using the Laplace's cofactor expansion along the first and $(m+1)$-th rows we obtain

$$
\operatorname{det} M(m)=\operatorname{det} M(1) \operatorname{det} M(m-1) .
$$

By induction the formula (14) implies that

$$
\operatorname{det} M(m)=(\operatorname{det} M(1))^{m} .
$$

Now it follows from (5), (6) and (7) that

$$
\Theta_{m}=\left(\theta_{1}\right)^{m}, \Theta_{-m}=\left(\theta_{-1}\right)^{m}
$$

which implies that the boundary conditions (3) are regular if (4) are regular.

By (10), (13) and (15), $\zeta_{1}$ and $\zeta_{2}$ are the roots of the equation

$$
\Theta_{-m} \zeta^{-m}+\Theta_{-m+1} \zeta^{m-1}+\ldots+\Theta_{m} \zeta^{m}=0
$$

with multiplicity $m$. Therefore it follows from Theorem 2 in p.123 of [7] that to each root $\zeta_{1}$ and $\zeta_{2}$ correspond $m$ sequences, denoted by

$\left\{\lambda_{k, 1}^{(1)}: k=N, N+1, \ldots\right\},\left\{\lambda_{k, 2}^{(1)}: k=N, N+1, \ldots\right\}, \ldots,\left\{\lambda_{k, m}^{(1)}: k=N, N+1, \ldots\right\}$ 
and

$\left\{\lambda_{k, 1}^{(2)}: k=N, N+1, \ldots\right\},\left\{\lambda_{k, 2}^{(2)}: k=N, N+1, \ldots\right\}, \ldots,\left\{\lambda_{k, m}^{(2)}: k=N, N+1, \ldots\right\}$

respectively, satisfying

$$
\lambda_{k, j}^{(1)}=\left(2 k \pi+\gamma_{1}\right)^{2}+O\left(k^{1-\frac{1}{m}}\right), \lambda_{k, j}^{(2)}=\left(2 k \pi+\gamma_{2}\right)^{2}+O\left(k^{1-\frac{1}{m}}\right)
$$

for $k=N, N+1, \ldots$ and $j=1,2, \ldots, m$, where $N \gg 1$.

Now to analyze the operators $L_{m}(0), L_{m}(C)$ and $L_{m}(Q)$, we introduce the following notations. To simplify the notations we omit the upper indices in $\rho_{n}^{(1)}(0), \rho_{n}^{(2)}(0), \lambda_{k, j}^{(1)}, \lambda_{k, j}^{(2)}$ (see (8) and (16)) and enumerate these eigenvalues in the following way

$$
\rho_{n}^{(1)}(0)=: \rho_{n}, \rho_{n}^{(2)}(0)=: \rho_{-n}, \lambda_{k, j}^{(1)}=: \lambda_{k, j}, \lambda_{k, j}^{(2)}=: \lambda_{-k, j}
$$

for $n>0$ and $k \geq N \gg 1$. We remark that there is one-to-one correspondence between the eigenvalues (counting with multiplicities) of the operator $L_{1}(0)$ and integers which preserve asymptotic (8). This statement can easily be proved in a standard way by using Rouche's theorem (we omit the proof of this fact, since it is used only to simplify the notations). Denote the normalized eigenfunction of the operator $L_{1}(0)$ corresponding to the eigenvalue $\rho_{n}$ by $\varphi_{n}$. Clearly,

$$
\varphi_{n, 1}=\left(\varphi_{n}, 0,0, \ldots 0\right)^{T}, \varphi_{n, 2}=\left(0, \varphi_{n}, 0, \ldots 0\right)^{T}, \ldots, \varphi_{n, m}=\left(0,0, \ldots 0, \varphi_{n}\right)^{T}
$$

are the eigenfunctions of the operator $L_{m}(0)$ corresponding to the eigenvalue $\rho_{n}$. Similarly,

$$
\varphi_{n, 1}^{*}=\left(\varphi_{n}^{*}, 0,0, \ldots 0\right)^{T}, \varphi_{n, 2}^{*}=\left(0, \varphi_{n}^{*}, 0, \ldots 0\right)^{T}, \ldots, \varphi_{n, m}^{*}=\left(0,0, \ldots 0, \varphi_{n}^{*}\right)^{T}
$$

are the eigenfunctions of the operator $L_{m}^{*}(0)$ corresponding to the eigenvalue $\overline{\rho_{n}}$, where $\varphi_{n}^{*}$ is the eigenfunction of $L_{1}^{*}(0)$ corresponding to the eigenvalue $\overline{\rho_{n}}$.

Since the boundary conditions (4) are strongly regular, all eigenvalues of sufficiently large modulus of $L_{1}(q)$ are simple (see the end of Theorem 2 of p.65, [7]). Therefore, there exists $n_{0}$ such that the eigenvalues $\rho_{n}$ of $L_{1}(0)$ are simple for $|n|>n_{0}$. However, the operator $L_{1}(0)$ may have associated functions $\varphi_{n}^{(1)}, \varphi_{n}^{(2)}, \ldots, \varphi_{n}^{(t(n))}$ corresponding to the eigenfunction $\varphi_{n}$ for $|n| \leq n_{0}$. Then, it is not hard to see that $L_{m}(0)$ has associated functions

$\varphi_{n, 1, p}=\left(\varphi_{n}^{(p)}, 0,0, \ldots 0\right)^{T}, \varphi_{n, 2, p}=\left(0, \varphi_{n}^{(p)}, 0, \ldots 0\right)^{T}, \ldots, \varphi_{n, m, p}=\left(0,0, \ldots 0, \varphi_{n}^{(p)}\right)^{T}$,

for $p=1,2, \ldots, t(n)$ corresponding to $\rho_{n}$ for $|n| \leq n_{0}$, that is,

$$
\begin{aligned}
& \left(L_{m}(0)-\rho_{n}\right) \varphi_{n, i, 0}=0, \\
& \left(L_{m}(0)-\rho_{n}\right) \varphi_{n, i, p}=\varphi_{n, i, p-1}, \quad p=1,2, \ldots, t(n),
\end{aligned}
$$

where $\varphi_{n, i, 0}(x)=: \varphi_{n, i}(x)$. Since the system of the root functions of $L_{1}(0)$ forms Riesz basis in $L_{2}(0,1)$ (see [6]), the system

$$
\left\{\varphi_{n, i, p}: n \in \mathbb{Z}, i=1,2, \ldots, m, p=1,2, \ldots, t(n)\right\}
$$


forms a Riesz basis in $L_{2}^{m}(0,1)$. The system,

$$
\left\{\varphi_{n, i, p}^{*}: n \in \mathbb{Z}, i=1,2, \ldots, m, p=1,2, \ldots, t(n)\right\}
$$

which is biorthogonal to $\left\{\varphi_{n, i, p}\right\}$ is the system of the eigenfunctions and the associated functions of the adjoint operator $L_{m}^{*}(0)$. Clearly, (21) can be constructed by repeating the construction of (20) and replacing everywhere $\varphi_{n}$ by $\varphi_{n}^{*}$. Thus

$$
\begin{aligned}
& \left(L_{m}^{*}(0)-\overline{\rho_{n}}\right) \varphi_{n, i, 0}^{*}=0, \\
& \left(L_{m}^{*}(0)-\overline{\rho_{n}}\right) \varphi_{n, i, p}^{*}=\varphi_{n, i, p-1}^{*}, \quad p=1,2, \ldots, t(n) .
\end{aligned}
$$

To prove the main results, we need the following properties of the eigenfunctions $\varphi_{n}$ and $\varphi_{n}^{*}$.

Proposition 1 If the boundary conditions (4) are strongly regular then there exists a positive constant $M$ such that

$$
\begin{gathered}
\sup _{x \in[0,1]}\left|\varphi_{n}(x)\right| \leq M, \sup _{x \in[0,1]}\left|\varphi_{n}^{*}(x)\right| \leq M, \\
\sup _{x \in[0,1]}\left|\varphi_{n, i, p}(x)\right| \leq M, \sup _{x \in[0,1]}\left|\varphi_{n, i, p}^{*}(x)\right| \leq M,
\end{gathered}
$$

for all $n, i, p$, where $\varphi_{n}^{*}$ is the eigenfunctions of $L_{1}^{*}(0)$, satisfying

$$
\left(\varphi_{n}, \varphi_{n}^{*}\right)=1
$$

for $|n|>n_{0}$. Moreover, the following asymptotic formulas hold

$$
\begin{aligned}
& \overline{\varphi_{n}^{*}(x)} \varphi_{n}(x)=1+A_{1} e^{i\left(4 \pi n+2 \gamma_{1}\right) x}+B_{1} e^{-i\left(4 \pi n+2 \gamma_{1}\right) x}+O\left(\frac{1}{n}\right), n>0, \\
& \overline{\varphi_{n}^{*}(x)} \varphi_{n}(x)=1+A_{2} e^{i\left(4 \pi n+2 \gamma_{2}\right) x}+B_{2} e^{-i\left(4 \pi n+2 \gamma_{2}\right) x}+O\left(\frac{1}{n}\right), n<0,
\end{aligned}
$$

where $A_{j}$ and $B_{j}$ for $j=1,2$ are constants.

Proof. It is well-known that (see p. 62-63 of [7]) if the boundary conditions (4) are strongly regular then these are either Dirichlet boundary conditions: $y(1)=y(0)=0$ or

$$
\begin{aligned}
& y^{\prime}(0)+\alpha_{11} y(0)+\alpha_{12} y(1)=0, \\
& y^{\prime}(1)+\alpha_{21} y(0)+\alpha_{22} y(1)=0
\end{aligned}
$$

or

$$
\begin{aligned}
a_{1} y^{\prime}(0)+b_{1} y^{\prime}(1)+a_{0} y(0)+b_{0} y(1) & =0 \\
c_{0} y(0)+d_{0} y(1) & =0 .
\end{aligned}
$$


In the case of Dirichlet boundary conditions we have

$$
\varphi_{n}(x)=\varphi_{n}^{*}(x)=\sqrt{2} \sin 2 n x, \varphi_{-n}(x)=\varphi_{-n}^{*}(x)=\sqrt{2} \sin (2 n+1) x,
$$

where $n=1,2, \ldots$ In case (28) using the well known expression for eigenfunction

$$
\left|\begin{array}{cc}
e^{i \rho_{n} x} & e^{-i \rho_{n} x} \\
U_{1}\left(e^{i \rho_{n} x}\right) & U_{1}\left(e^{-i \rho_{n} x}\right)
\end{array}\right|
$$

and (8), (17), and also taking into account that $\gamma_{1}=0$ and $\gamma_{2}=\pi$ (see (6) and (11)), we obtain

$$
\varphi_{n}(x)=\sqrt{2} \cos 2 n x+O\left(\frac{1}{n}\right), \varphi_{-n}(x)=\sqrt{2} \cos (2 n+1) x+O\left(\frac{1}{n}\right)
$$

and

$$
\varphi_{n}^{*}(x)=\sqrt{2} \cos 2 n x+O\left(\frac{1}{n}\right), \varphi_{-n}^{*}(x)=\sqrt{2} \cos (2 n+1) x+O\left(\frac{1}{n}\right) .
$$

In the same way in case (29) we get the formulas

$$
\begin{aligned}
& \varphi_{n}(x)=a^{+} e^{i\left(2 \pi n+\gamma_{1}\right) x}+b^{+} e^{-i\left(2 \pi n+\gamma_{1}\right) x}+O\left(\frac{1}{n}\right), \\
& \varphi_{n}^{*}(x)=c^{+} e^{i\left(2 \pi n+\gamma_{1}\right) x}+d^{+} e^{-i\left(2 \pi n+\gamma_{1}\right) x}+O\left(\frac{1}{n}\right)
\end{aligned}
$$

for $n>0$ and

$$
\begin{aligned}
& \varphi_{n}(x)=a^{-} e^{i\left(2 \pi n+\gamma_{2}\right) x}+b^{-} e^{-i\left(2 \pi n+\gamma_{2}\right) x}+O\left(\frac{1}{n}\right), \\
& \varphi_{n}^{*}(x)=c^{-} e^{i\left(2 \pi n+\gamma_{2}\right) x}+d^{-} e^{-i\left(2 \pi n+\gamma_{2}\right) x}+O\left(\frac{1}{n}\right)
\end{aligned}
$$

for $n<0$. Thus in any case inequality (24) holds. Equality (25) follows from (24) and equality (27) follows from (26), (30)-(34).

As it is noted in the introduction, we obtain asymptotic formulas for the eigenvalues and eigenfunctions of $L_{m}(Q)$ in term of the eigenvalues and eigenfunctions of $L_{m}(C)$. Therefore first we analyze the eigenvalues and eigenfunctions of $L_{m}(C)$. Suppose that the matrix $C$ has $m$ simple eigenvalues $\mu_{1}, \mu_{2}, \ldots, \mu_{m}$. The normalized eigenvector corresponding to the eigenvalue $\mu_{j}$ is denoted by $v_{j}$. In these notations the eigenvalues and eigenfunctions of $L_{m}(C)$ are

$$
\mu_{k, j}=\rho_{k}+\mu_{j} \quad \& \quad \Phi_{k, j}(x)=v_{j} \varphi_{k}(x)
$$

respectively. Similarly, the eigenvalues and eigenfunctions of $\left(L_{m}(C)\right)^{*}$ are $\overline{\mu_{k, j}}$, $\Phi_{k, j}^{*}(x)=v_{j}^{*} \varphi_{k}^{*}$, where $v_{j}^{*}$ is the eigenvector of $C^{*}$ corresponding to $\overline{\mu_{j}}$ such that $\left(v_{j}^{*}, v_{j}\right)=1$. To obtain the asymptotic formulas for the eigenvalues and eigenfunctions of $L_{m}(Q)$, we use the following formula

$$
\left(\lambda_{k, j}-\mu_{k, i}\right)\left(\Psi_{k, j}, \Phi_{k, i}^{*}\right)=\left((Q-C) \Psi_{k, j}, \Phi_{k, i}^{*}\right)
$$


obtained from

$$
L_{m}(Q) \Psi_{k, j}(x)=\lambda_{k, j} \Psi_{k, j}(x)
$$

by multiplying both sides of (37) with $\Phi_{k, i}^{*}(x)$ and using $L_{m}(Q)=L_{m}(C)+$ $(Q-C)$. To prove that $\lambda_{k, j}$ is close to $\mu_{k, j}$, we first show that the right-hand side of (36) is a small number for all $j$ and $i$ (see Lemma 1) and then we prove that for each eigenfunction $\Psi_{k, j}$ of $L_{m}(Q)$, where $|k| \geq N$, there exists a root function of $\left.\left(L_{m}(C)\right)\right)^{*}$ denoted by $\Phi_{k, j}^{*}$ such that $\left(\Psi_{k, j}, \Phi_{k, j}^{*}\right)$ is a number of order 1 (see Lemma 2). Before the proof of these lemmas, we need the following preparations: Multiplying both sides of (37) by $\varphi_{n, i, 0}^{*}$, using $L_{m}(Q)=L_{m}(0)+Q$ and (22) we get

$$
\begin{gathered}
\left(\lambda_{k, j}-\rho_{n}\right)\left(\Psi_{k, j}, \varphi_{n, i, 0}^{*}\right)=\left(Q \Psi_{k, j}, \varphi_{n, i, 0}^{*}\right), \\
\left(\Psi_{k, j}, \varphi_{n, i, 0}^{*}\right)=\frac{\left(Q \Psi_{k, j}, \varphi_{n, i, 0}^{*}\right)}{\lambda_{k, j}-\rho_{n}}
\end{gathered}
$$

for $i, j=1,2, \ldots, m$ and $\lambda_{k, j} \neq \rho_{n}$. Now multiplying (37) by $\varphi_{n, i, 1}^{*}$ and using (23), (38), we get

$$
\begin{gathered}
\left(\lambda_{k, j}-\rho_{n}\right)\left(\Psi_{k, j}, \varphi_{n, i, 1}^{*}\right)=\left(Q \Psi_{k, j}, \varphi_{n, i, 1}^{*}\right)+\frac{\left(Q \Psi_{k, j}, \varphi_{n, i, 0}^{*}\right)}{\lambda_{k, j}-\rho_{n}}, \\
\left(\Psi_{k, j}, \varphi_{n, i, 1}^{*}\right)=\frac{\left(Q \Psi_{k, j}, \varphi_{n, i, 1}^{*}\right)}{\lambda_{k, j}-\rho_{n}}+\frac{\left(Q \Psi_{k, j}, \varphi_{n, i, 0}^{*}\right)}{\left(\lambda_{k, j}-\rho_{n}\right)^{2}} .
\end{gathered}
$$

In this way one can deduce the formulas

$$
\left(\Psi_{k, j}, \varphi_{n, i, s}^{*}\right)=\sum_{p=0}^{s} \frac{\left(Q \Psi_{k, j}, \varphi_{n, i, p}^{*}\right)}{\left(\lambda_{k, j}-\rho_{n}\right)^{s+1-p}}
$$

for $s=0,1, \ldots, t(n)$.

Since, $\left(Q \Psi_{k, j}, \varphi_{n, i, p}^{*}\right)=\left(\Psi_{k, j}, Q^{*} \varphi_{n, i, p}^{*}\right),\left\|\Psi_{k, j}\right\|=1$, and the entries of $Q$ are the elements of $L_{2}(0,1)$, it follows from (25) and Cauchy-Schwarz inequality that there exists a positive constant $c_{1}$ such that

$$
\left|\left(Q \Psi_{k, j}, \varphi_{n, i, p}^{*}\right)\right|<c_{1} .
$$

In the subsequent estimates we denote by $c_{m}$ for $m=1,2, \ldots$, the positive constants whose exact value are inessential. On the other hand, it follows from (8), (16) and (17) that if $k$ is a sufficiently large number then

$$
\begin{gathered}
\left|\lambda_{k, j}-\rho_{p}\right|>c_{2} k^{2}, \forall p \leq n_{0}, \\
\left|\lambda_{k, j}-\rho_{p}\right|>c_{3}|| p|-| k||(|p|+|k|), \forall p \neq \pm k,
\end{gathered}
$$

and

$$
\left|\lambda_{k, j}-\rho_{-k}\right|>c_{4}|k|
$$


Therefore by (38)-(43) we have

$$
\begin{gathered}
\left|\left(\Psi_{k, j}, \varphi_{p, q, s}^{*}\right)\right|<c_{5} k^{-2}, \forall p \leq n_{0}, \\
\left|\left(\Psi_{k, j}, \varphi_{p, q}^{*}\right)\right| \leq \frac{c_{6}}{|| p|-| k||(|p|+|k|)}, \quad\left|\left(\Psi_{k, j}, \varphi_{-k, q}^{*}\right)\right| \leq \frac{c_{7}}{|k|}
\end{gathered}
$$

for all $|k| \gg 1, p \neq \pm k, s=0,1, \ldots, t(p)$ and $q, j=1,2, \ldots, m$.

Now, we are ready to prove the lemmas.

Lemma 1 For any $i=1,2, \ldots, m$ and $j=1,2, \ldots, m$ the following estimation holds

$$
\left(\Psi_{k, j},\left(Q^{*}-C^{*}\right) \Phi_{k, i}^{*}\right)=O\left(\alpha_{k}\right)+O\left(\frac{\ln |k|}{k}\right),
$$

where $\alpha_{k}=\max \left\{\left|b_{s, i, 2 k, r}^{+}\right|,\left|b_{s, i, 2 k, r}^{-}\right|: s, i=1,2, \ldots, m ; r=1,2\right\}$,

$$
b_{s, i, 2 k, r}^{ \pm}=\int_{0}^{1} b_{s, i}(x) e^{ \pm i\left(4 \pi k+2 \gamma_{r}\right) x}
$$

$b_{s, i} \in L_{2}(0,1)$ are the entries of the matrix $Q$, and $\gamma_{r}$ is defined in (9).

Proof. Since $\Phi_{k, i}^{*}(x)=v_{i}^{*} \varphi_{k}^{*}(x)$, it is enough to prove that

$$
\left(\Psi_{k, j},\left(Q^{*}-C^{*}\right) \varphi_{k, s}^{*}\right)=O\left(\alpha_{k}\right)+O\left(\frac{\ln |k|}{k}\right)
$$

for $s=1,2, \ldots, m$. The decomposition of $\left(Q^{*}-C^{*}\right) \varphi_{k, s}^{*}$ by the basis (21) has the form

$$
\begin{gathered}
\left(Q^{*}-C^{*}\right) \varphi_{k, s}^{*}=\sum_{q=1}^{m} \sum_{p:|p| \leq n_{0}} \sum_{v=1}^{t(p)} c(k, s, p, q, v) \varphi_{p, q, v}^{*}+ \\
\sum_{q=1,2, \ldots m} \sum_{p:|p|>n_{0}}^{\infty}\left(\left(Q^{*}-C^{*}\right) \varphi_{k, s}^{*}, \varphi_{p, q}\right) \varphi_{p, q}^{*}
\end{gathered}
$$

Therefore

$$
\begin{array}{r}
\left(\Psi_{k, j},\left(Q^{*}-C^{*}\right) \varphi_{k, s}^{*}\right)=\sum_{q=1}^{m} \sum_{p:|p| \leq n_{0}} \sum_{v=1}^{t(p)} \overline{c(k, s, p, q, v)}\left(\Psi_{k, j}, \varphi_{p, q, v}^{*}\right)+ \\
\sum_{q=1}^{m} \sum_{p:|p|>n_{0}} \overline{\left(\left(Q^{*}-C^{*}\right) \varphi_{k, s}^{*}, \varphi_{p, q}\right)}\left(\Psi_{k, j}, \varphi_{p, q}^{*}\right)
\end{array}
$$

Since $c(k, s, p, q, v)=O(1)$, by (44) the first summation of the right hand side of $(49)$ is $O\left(k^{-2}\right)$.

Now let us estimate the second summation $S$ of the right hand side of (49). It can be written in the form

$$
S=S_{1}+S_{2}
$$


where

$$
\begin{aligned}
& S_{1}=\sum_{q=1}^{m} \overline{\left(\left(Q^{*}-C^{*}\right) \varphi_{k, s}^{*}, \varphi_{k, q}\right)}\left(\Psi_{k, j}, \varphi_{k, q}^{*}\right), \\
& S_{2}=\sum_{q=1}^{m} \sum_{p \neq k} \overline{\left(\left(Q^{*}-C^{*}\right) \varphi_{k, s}^{*}, \varphi_{p, q}\right)}\left(\Psi_{k, j}, \varphi_{p, q}^{*}\right) .
\end{aligned}
$$

Using (18), (19), (25) and (27), one can easily verify that

$$
S_{1}=O\left(\alpha_{k}\right)+O\left(\frac{1}{k}\right)
$$

On the other hand, by (45), we have

$$
S_{2}=O\left(\frac{\ln |k|}{k}\right)
$$

Therefore, (48) follows from (49)-(52). The lemma is proved.

Lemma 2 For each eigenfunction $\Psi_{k, j}$ of $L_{m}(Q)$, where $|k| \geq N$, there exists an eigenfunction of $\left(L_{m}(C)\right)^{*}$ denoted by $\Phi_{k, j}^{*}$ such that

$$
\left|\left(\Psi_{k, j}, \Phi_{k, j}^{*}\right)\right|>c_{8} .
$$

Proof. Since (20) is a Riesz basis of $L_{2}^{m}[0,1]$, we have

$$
\Psi_{k, j}=\sum_{q=1}^{m} \sum_{p:|p| \leq n_{0}} \sum_{v=1}^{t(p)} c(k, j, p, q, v) \varphi_{p, q, v}+\sum_{q=1}^{m} \sum_{p:|p|>n_{0}}\left(\Psi_{k, j}, \varphi_{p, q}^{*}\right) \varphi_{p, q} .
$$

It follows from (25) and (45) that

$$
\sum_{q=1}^{m} \sum_{\substack{p \neq k \\|p|>n_{0}}}\left\|\left(\Psi_{k, j}, \varphi_{p, q}^{*}\right) \varphi_{p, q}\right\|=O\left(\frac{\ln |k|}{k}\right) .
$$

On the other hand, arguing as in the estimation for the first summation of (49) we get

$$
\sum_{q=1}^{m} \sum_{p:|p| \leq n_{0}} \sum_{j=1}^{t(p)}\left\|c(k, s, p, q, j) \varphi_{p, q, j}\right\|=O\left(\frac{1}{k^{2}}\right) .
$$

Therefore using (55), (54), we obtain

$$
\Psi_{k, j}=\sum_{q=1}^{m}\left(\Psi_{k, j}, \varphi_{k, q}^{*}\right) \varphi_{k, q}+O\left(\frac{\ln |k|}{k}\right)
$$

Since $\left\{\varphi_{k, 1}, \varphi_{k, 2}, \ldots ., \varphi_{k, m}\right\}$ is orthonormal system and $\left\|\Psi_{k, j}\right\|=1$, there exists an index $q$ such that

$$
\left|\left(\Psi_{k, j}, \varphi_{k, q}^{*}\right)\right|>c_{9} .
$$


On the other hand

$$
\varphi_{k, q}^{*}=\sum_{j=1}^{m}\left(\varphi_{k, q}^{*}, \Phi_{k, j}\right) \Phi_{k, j}^{*}
$$

because $\Phi_{k, j}^{*}=v_{j}^{*} \varphi_{k}^{*}$, and the vectors $v_{j}^{*}, j=1,2, \ldots m$ form a basis in $\mathbb{C}^{m}$. Now, using (58) in (57), we get the proof of the lemma.

Theorem 2 Suppose that all eigenvalues $\mu_{1}, \mu_{2}, \ldots, \mu_{m}$ of the matrix $C$ are simple. Then, there exists a number $N$ such that all eigenvalues $\lambda_{k, 1}, \lambda_{k, 2}, \ldots, \lambda_{k, m}$ of $L_{m}(Q)$ for $|k| \geq N$ are simple and satisfy the asymptotic formula

$$
\lambda_{k, j}=\mu_{k, j}+O\left(\alpha_{k}\right)+O\left(\frac{\ln |k|}{k}\right),
$$

where $\mu_{k, j}$ is the eigenvalue of $L_{m}(C)$ and $\alpha_{k}$ is defined in Lemma 1. The normalized eigenfunction $\Psi_{k, j}(x)$ of $L_{m}(Q)$ corresponding to $\lambda_{k, j}$ satisfies

$$
\Psi_{k, j}(x)=\Phi_{k, j}(x)+O\left(\alpha_{k}\right)+O\left(\frac{\ln |k|}{k}\right),
$$

where $\Phi_{k, j}(x)$ is the normalized eigenfunction of $L_{m}(C)$ corresponding to $\mu_{k, j}$. The root functions of $L_{m}(Q)$ form a Riesz basis in $L_{2}^{m}(0,1)$.

Proof. In (36) replacing $i$ by $j$, and then dividing the both sides of the obtained equality by $\left(\Psi_{k, j}, \Phi_{k, j}^{*}\right)$ and using Lemma 1 and Lemma 2, we see all large eigenvalues of $L_{m}(Q)$ lie in $r_{k}$ neighborhood of the eigenvalues $\mu_{k, j}$ for $|k| \geq N, j=1,2, \ldots, m$ of $L_{m}(C)$, where

$$
r_{k}=O\left(\alpha_{k}\right)+O\left(\frac{\ln |k|}{k}\right) .
$$

Now we prove that these eigenvalues are simple. Let $\lambda_{k, j}$ be an eigenvalue of $L_{m}(Q)$ lying in $\frac{1}{2} a_{j}$ neighborhood of $\mu_{k, j}=\rho_{k}+\mu_{j}$ (see (35)), where $a_{j}=$ $\min _{i \neq j}\left|\mu_{j}-\mu_{i}\right|$. Then, by triangle inequality

$$
\left|\lambda_{k, j}-\mu_{k, i}\right|>\left|\mu_{k, j}-\mu_{k, i}\right|-\left|\lambda_{k, j}-\mu_{k, j}\right| \geq a_{j}-\frac{1}{2} a_{j}=\frac{1}{2} a_{j}
$$

for $i \neq j$. Therefore using (36) and Lemma 1 we get

$$
\left(\Psi_{k, j}, \Phi_{k, i}^{*}\right)=O\left(\alpha_{k}\right)+O\left(\frac{\ln |k|}{k}\right)
$$

for $i \neq j$. This and (56) imply that (60) holds for any normalized eigenfunction corresponding to $\lambda_{k, j}$, since

$$
\operatorname{span}\left\{\varphi_{k, 1}, \varphi_{k, 2}, \ldots, \varphi_{k, m}\right\}=\operatorname{span}\left\{\Phi_{k, 1}, \Phi_{k, 2}, \ldots, \Phi_{k, m}\right\} .
$$

Using this, let us prove that $\lambda_{k, j}$ is a simple eigenvalue. Suppose to the contrary that $\lambda_{k, j}$ is a multiple eigenvalue. If there are two linearly independent eigenfunctions corresponding to $\lambda_{k, j}$, then one can find two orthogonal eigenfunctions 
satisfying (60), which is impossible. Hence there exists a unique eigenfunction $\Psi_{k, j}$ corresponding to $\lambda_{k, j}$. If there exists an associated function $\Psi_{k, j, 1}$ belonging to the eigenfunction $\Psi_{k, j}$, then

$$
\left(L_{m}(Q)-\lambda_{k, j}\right) \Psi_{k, j, 1}(x)=\Psi_{k, j}(x) .
$$

Multiplying both sides of this equality by $\Psi_{k, j}^{*}(x)$, where $\Psi_{k, j}^{*}(x)$ is the normalized eigenfunction of $\left(L_{m}(Q)\right)^{*}$ corresponding to the eigenvalue $\overline{\lambda_{k, j}}$, we obtain

$$
\left.\left(\Psi_{k, j}, \Psi_{k, j}^{*}\right)=\left(\Psi_{k, i, 1},\left(\left(L_{m}(Q)\right)^{*}-\overline{\lambda_{k, j}}\right)\right) \Psi_{k, j}^{*}\right)=0 .
$$

Since the proved statements are also applicable for the adjoint operator $\left(L_{m}(Q)\right)^{*}$, formula (60) holds for this operator too, that is, we have

$$
\Psi_{k, j}^{*}(x)=\Phi_{k, j}^{*}(x)+O\left(\alpha_{k}\right)+O\left(\frac{\ln |k|}{k}\right) .
$$

This formula, (60) and the obvious relation $\left(\Phi_{k, j}, \Phi_{k, j}^{*}\right)=1$ contradict with (62). Thus, $\lambda_{k, j}$ is a simple eigenvalue.

We proved that all large eigenvalues of $L_{m}(Q)$ lie in the disk

$$
\Delta_{k, j}=\left\{z:\left|z-\mu_{k, j}\right|<r_{k}\right\}
$$

for $|k| \geq N, j=1,2, \ldots, m$, where $r_{k}$ is defined in (61). Clearly, the disks $\Delta_{k, j}$ for $j=1,2, \ldots, m$ and $|k| \geq N$ are pairwise disjoint. Let us prove that each of these disks does not contain more than one eigenvalue of $L_{m}(Q)$. Suppose to the contrary that, two different eigenvalues $\Lambda_{1}$ and $\Lambda_{2}$ lie in $\Delta_{k, j}$. Then it has already been proven that these eigenvalues are simple and the corresponding eigenfunctions $\Psi_{1}$ and $\Psi_{2}$ satisfy

$$
\Psi_{p}(x)=\Phi_{k, j}(x)+O\left(\alpha_{k}\right)+O\left(\frac{\ln |k|}{k}\right)
$$

for $p=1,2$. Similarly, the eigenfunctions $\Psi_{1}^{*}$ and $\Psi_{2}^{*}$ of $\left(L_{m}(Q)\right)^{*}$ corresponding to the eigenvalues $\overline{\Lambda_{1}}$ and $\overline{\Lambda_{2}}$ satisfy

$$
\Psi_{p}^{*}(x)=\Phi_{k, j}^{*}(x)+O\left(\alpha_{k}\right)+O\left(\frac{\ln |k|}{k}\right) .
$$

for $p=1,2$. Since $\Lambda_{1} \neq \Lambda_{2}$, we have

$$
0=\left(\Psi_{1}, \Psi_{2}^{*}\right)=1+O\left(\alpha_{k}\right)+O\left(\frac{\ln |k|}{k}\right)
$$

which is impossible. Hence the pairwise disjoint disks $\Delta_{k, 1}, \Delta_{k, 2}, \ldots, \Delta_{k, m}$, where $|k| \geq N$, contain $m$ eigenvalues of $L_{m}(Q)$ and each of these disks does not contain more than one eigenvalue. Therefore, there exists a unique eigenvalue $\lambda_{k, j}$ of $L_{m}(Q)$ lying in $\Delta_{k, j}$, where $j=1,2, \ldots, m$ and $|k| \geq N$. Thus the eigenvalues $\lambda_{k, j}$ for $|k| \geq N$ are simple and the formulas (59) and (60) hold. 
It remains to prove that the root functions of $L_{m}(Q)$ form a Riesz basis in $L_{2}^{m}(0,1)$. For this, let us prove that for $f \in L_{2}^{m}(0,1)$, the following series is convergent

$$
\sum_{j=1}^{m} \sum_{k=N+1}^{\infty}\left|\left(f, \Psi_{k, j}\right)\right|^{2}<\infty
$$

where $N$ is a large positive number. By the asymptotic formula (60), we have

$$
\sum_{k=N+1}^{\infty}\left|\left(f, \Psi_{k, j}\right)\right|^{2} \leq 3\left(\sum_{k=N+1}^{\infty}\left|\left(f, \Phi_{k, j}\right)\right|^{2}+\sum_{k=N+1}^{\infty}\left|\left(f, g_{k}\right)\right|^{2}+\sum_{k=N+1}^{\infty}\left|\left(f, h_{k}\right)\right|^{2}\right)
$$

where $\left\|g_{k}\right\|=O\left(\alpha_{k}\right)$ and $\left\|h_{k}\right\|=O\left(\frac{\ln |k|}{k}\right)$. The first series in the right side of (65) converges, since the root functions of $L_{m}(C)$ is a Riesz basis in $L_{2}^{m}(0,1)$. Using the Cauchy-Schwarz inequality we get

$$
\sum_{k=N+1}^{\infty}\left|\left(f, g_{k}\right)\right|^{2} \leq c_{10}\|f\|^{2} \sum_{k=N+1}^{\infty}\left|\alpha_{k}\right|^{2}
$$

On the other hand, using the definition of $\alpha_{k}$ (see Lemma 1) and taking into account that the entries of the matrix $Q$ are the element of $L_{2}(0,1)$, we obtain

$$
\sum_{k=N+1}^{\infty}\left|\alpha_{k}\right|^{2}<\infty
$$

Therefore, by (66), the second series in the right side of (65) converges too. In the same way, we prove that the third series in the right side of (65) converges. Thus, the series of the left-hand side of (65) converges, that is, (64) is proved. By Bari's definition (see [2], chap. 6), this implies that the system of eigenfunctions of the operator under consideration is Bessel. Since the system of root functions of the adjoint operator has the asymptotics (63), in the same way, we obtain that it is also Bessel. Moreover, the equality

$$
\left(\Psi_{k, j}, \Psi_{k, j}^{*}\right)=1+O\left(\alpha_{k}\right)+O\left(\frac{\ln |k|}{k}\right)
$$

(see (60) and (63)) implies that the system of the root functions of $\left(L_{m}(Q)\right)^{*}$, which is biorthogonal to the system of the root functions of $L_{m}(Q)$, is also Bessel. As it is noted in $[11,12]$, the system of root functions of the operators $L_{m}(Q)$ and $\left(L_{m}(Q)\right)^{*}$ are complete in the space $L_{2}^{m}(0,1)$. These arguments and Bari's theorem in p.310 of [2] (if two biorthogonal systems are complete and Bessel, then they both are Riesz bases) conclude the proof of the theorem.

\section{References}

[1] R. Carlson, "Large Eigenvalues and Trace Formulas for Matrix SturmLiouville Problems", SIAM Journal on Mathematical Analysis 30 (5), 949962 (1999). 
[2] I. T. Goghberg and M. G. Krein, Introduction to the Theory of Linear Nonself-adjoint Operators ( AMS, Providence, 1969).

[3] L. M. Luzhina, "Regular Spectral Problems in the Space of Vectorfunctions", Moscow Univ. Math. Mech. Bull. 1 (1), 31-35 (1988).

[4] F. G. Maksudov, O. A. Veliev, "Nonself-adjoint Differential Operators in the Space of Vector-functions with Periodic Coefficients, Soviet Math. Dokl. 23, 475-478 (1981).

[5] F. G. Maksudov, O. A. Veliev, "Spectral Analysis of Differential Operators with Periodic Matrix Coefficients", Differentsial'nye Uravneniya 25 (3), 271-277 (1989).

[6] V. P. Mikhailov, On Riesz basis in $L^{2}(0,1)$, Dokl. Akad. Nauk. SSSR 144 (1962), 981-84.

[7] M. A. Naimark, Linear Differential Operators ( Frederick Ungar Pobl. Co. New York, 1967, 1968).

[8] A. A. Shkalikov, "On the Riesz basis property of the root vectors of ordinary differential operators," Uspekhi Mat. Nauk 34 (5), 235-236 (1979) [RussianMath. Surveys 34 (5), 249-250 (1979)].

[9] A. A. Shkalikov, "On the basis property of the eigenfunctions and associated functions of ordinary differential operators with integral boundary conditions," VestnikMoskov. Univ. Ser. I Mat. Mekh.,No. 6, 12-21 (1982).

[10] A. A. Shkalikov, "Boundary-value problems for ordinary differential equations with spectral parameter in theboundary conditions," Trudy Sem. Petrovsk., No. 9, 190-229 (1983).

[11] A. A. Shkalikov, "On the Riesz basis property of the eigenfunctions and associated functions of ordinary differential operators in the space of vector functions," in Functional Analysis and Its Applications in Mechanics and Probability Theory (Izd.Moskov. Univ.,Moscow, 1984) [in Russian].

[12] A. A. Shkalikov, On Riesz Basisness of the Root Functions of Ordinary Differential Operators in the Space of Vector-valued Functions, "In book: Functional Analysis and its Applications in Mechanics and Probability". MSU, 1984

[13] O. A. Veliev, On the Nonself-adjoint Sturm-Liouville Operators with Matrix Potentials, Mathematical Notes, Vol.81, No. 3-4, pp.440-448 (2007).

[14] Veliev O.A. "On the Hill's operator with a matrix potential, Mathematische Nachrichten, 281, NO.9, p.1341-1350, 2008.

[15] O. A. Veliev, Uniform Convergence of the Spectral Expansion for a Differential Operator with Periodic Matrix Coefficients, Boundary Value Problems, Volume 2008, Article ID 628973, 22 pages, 2008. 
[16] O. A. Veliev, On the Differential Operators with Periodic Matrix Coefficients, Abstract and Applied Analysis, Volume 2009, Article ID 934905, (21pp), 2009.

[17] O. A. Veliev, On the Basis Property of the Root Functions of Differential Operators with Matrix Coefficients, Central European Journal of Mathematics, Vol. 9, (16pp), 2011. 